\title{
Highly Miniaturized Mircrostrip Antenna with Slots and a Superstrate for RFID Applications
}

\author{
Yiyang Yu and Atif Shamim \\ Electrical Engineering Program, CEMSE Division \\ King Abdullah University of Science and Technology \\ Thuwal, Saudi Arabia \\ Yiyang.yu@kaust.edu.sa; Atif.shamim@kaust.edu.sa
}

\begin{abstract}
RFID Tags, typically, require ultra-small antennas and at $900 \mathrm{MHz}$ most of the designs end up being meandered line wire antennas. These may not be suitable because such antennas are heavily influenced by the object on which they are placed. An antenna with a ground plane, such as a microstrip antenna, is a more suitable candidate in that scenario, however, typically micrsotrip patch antennas are large in size. In this paper, we introduce a highly miniaturized microstrip antenna with multiple slots and a superstrate with high-permittivity material. The size of the microstrip antenna at $900 \mathrm{MHz}$ is reduced by $87.5 \%$ as compared to a conventional rectangular microstrip patch antenna at $900 \mathrm{MHz}$. The gain of the miniaturized antenna is $0.36 \mathrm{dBi}$, achieving $40 \%$ efficiency, which is decent enough for short to medium range RFID applications.
\end{abstract}

Keywords-Microstrip antenna; Miniaturization; Slots; Superstrate; High-contrast dielectric

\section{INTRODUCTION}

Microstrip antenna offers a remarkable advantage that an antenna with ground plane is good to be placed on objects without influencing antenna performance. Microstrip antenna is usually used in portable devices. However, there is a tendency of size reduction recently. The size of the inner components of these devices must be miniaturized to reduce the entire volume. Miniaturization of microstrip antenna has become an important issue due to its role in portable devices.

Several techniques are proposed to reduce the size of a microstrip antenna. Planar area reduction can be achieved by multilayer meanderly folded shorted-patch structure. A. Holub introduced a structure with a footprint dimension $\lambda_{0} / 16$ [1]. But it gives no ideal gain. F. Farzami has proposed another approach on miniaturization with a compact magneto-dielectric substrate and achieved $65 \%$ size reduction[2]. A. Roy has proposed a novel technique to realize compact microstrip patch antenna with symmetrical strips in both non radiating side [3], however, the gain is low $(-1 \mathrm{dBi})$. S. Chen has reported a slot structure for GPS antenna with high-constant dielectric, $\varepsilon_{r}=9.8$, offering $60.8 \%$ size reduction with $1 \mathrm{dBi}$ gain [4]. H. T. Nguyen has introduced an $\mathrm{H}$-shape slot for microstrip patch miniaturization [5], achieving $87.9 \%$ size reduction, however, the gain has not been reported.

In this paper, we report microstrip patch antenna miniaturization through a combination of multiple slots and a high permittivity $\left(\varepsilon_{r}=10\right)$ superstarte. A size reduction of $87.5 \%$ is achieved as compared to the conventional microstrip patch antenna at $900 \mathrm{MHz}$, with acceptable gain and radiation efficiency for RFID applications.

\section{MINIATURIZED ANTENNA DESIGN}

Slot loading is commonly used in planar antenna miniaturization, especially microstrip antenna. For a conventional rectangular microstrip antenna, the current flows from one edge to the other edge (on the longer side). However, the current has to make a detour if slots are introduced on the patch surface, causing a longer electric length which results in a lower resonant frequency.

The slot structure is realized by etching slots perpendicular to the direction of the flowing current to extend the current path. In Fig. 1. (a), the geometry of the patch loaded with two notches and one slot is presented. The dimensions of the patch are 16.4 $\times 27.2 \mathrm{~mm}^{2}$, giving $87.5 \%$ size reduction compared to a rectangular patch antenna at $900 \mathrm{MHz}$. The slot in the middle is $5 \times 26.7 \mathrm{~mm}^{2}$. The two notches both are $3 \times 13.2 \mathrm{~mm}^{2}$. The ground plane in Fig. 1 is twice the size of patch for a decent performance of the antenna. The impedance matching of the antenna is achieved by a $\lambda / 4$ impedance transformer. The $50 \Omega$ wave-port used to simulate the antenna is placed $\lambda / 4$ away from the impedance transformer. Fig. 1. (b) shows the distribution of the surface current density on the radiation patch. It can be seen that the current detours dramatically due to the slots and notches on the patch surface.
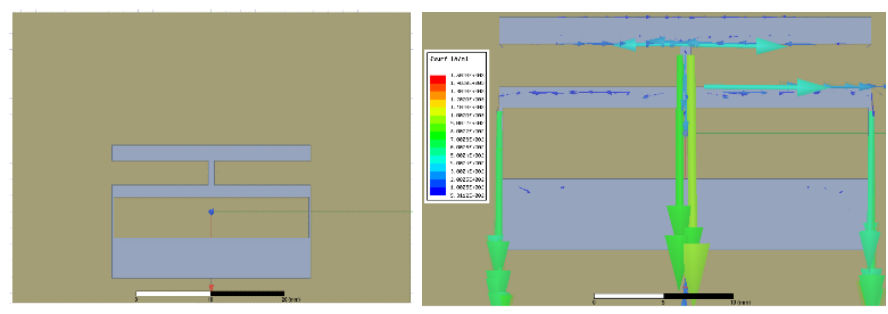

Fig. 1. HFSS simulation model for the slotted patch. (a) The slot sturcture of the top patch. (b) Vector of the surface current density on the patch

\section{SLOTTED ANTENNA WITH SUPERSTRATE}

For a conventional rectangular microstrip antenna, its length is approximately one-half of a free-space wavelength, when the substrate is air. It is well known that the dimensions of the patch antenna decrease as the relative permittivity of the dielectric 
material increases. In this paper, the thickness of the substrate $\mathrm{h}=9 \mathrm{~mm}$, dielectric constant $\varepsilon_{r}=10$, and the dielectric loss tangent $\delta=0.01$. In order to improve the radiation performance as well as reduce the size of the antenna, a superstrate is incorporated, shown in Fig. 2 [6]. The proposed $0.054 \lambda$ thick superstrate is realized with the same dielectric material as the substrate.

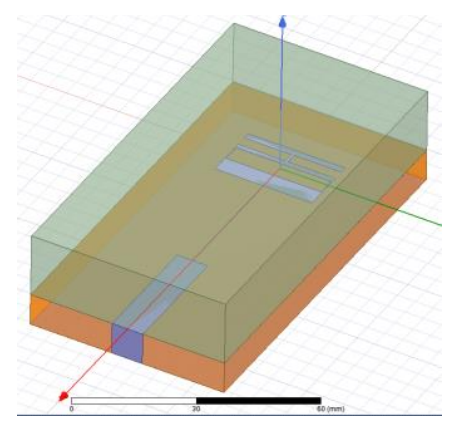

Fig. 2. Simulation model of the microstrip antenna with slots and superstrate.
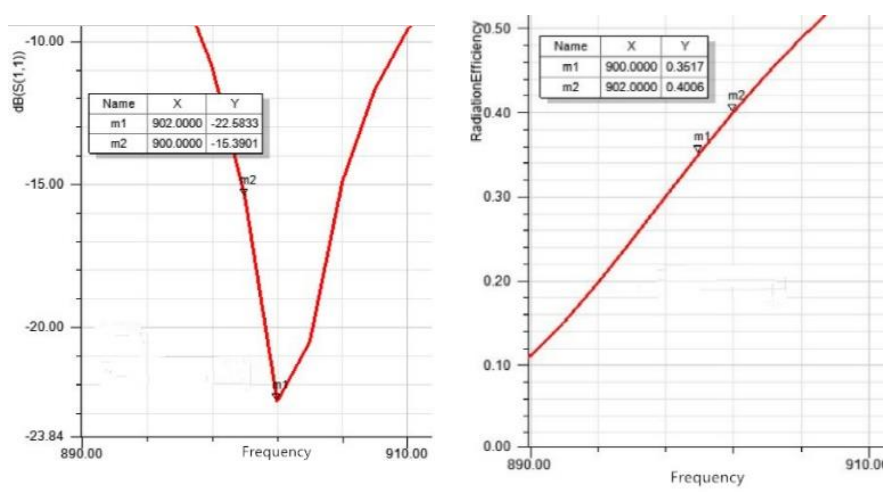

Fig. 3. HFSS simulation results. (a) $S_{11}$ vs. frequency. (b) Radiation efficiency vs. frequency

According to the simulation results in HFSS, shown in Fig. 3 . (a), the design resonates at $902 \mathrm{MHz}$ with $S_{11}=-22.58 \mathrm{~dB}$, and the impedance matching is decent at $900 \mathrm{MHz}$ still, $S_{11}=-$ $15.39 \mathrm{~dB}$. The radiation efficiency, as shown in Fig. 3. (b), reaches $40 \%$ at $902 \mathrm{MHz}$, and it is $35.17 \%$ at $900 \mathrm{MHz}$. Fig. 4 . shows the bore-sight maximum broad radiation pattern which is quite similar to the classical radiation pattern of a patch antenna. The gain is relatively low $(0.36 \mathrm{dBi})$, however this is expected due to high level of miniaturization. The proposed antenna design can be a competitive candidate for tiny RFID tags due to its ultra-small size, presence of ground plane which isolates the antenna from the objects on which the tag is placed, and decent radiation performance which is suitable for short to medium range RFID applications.

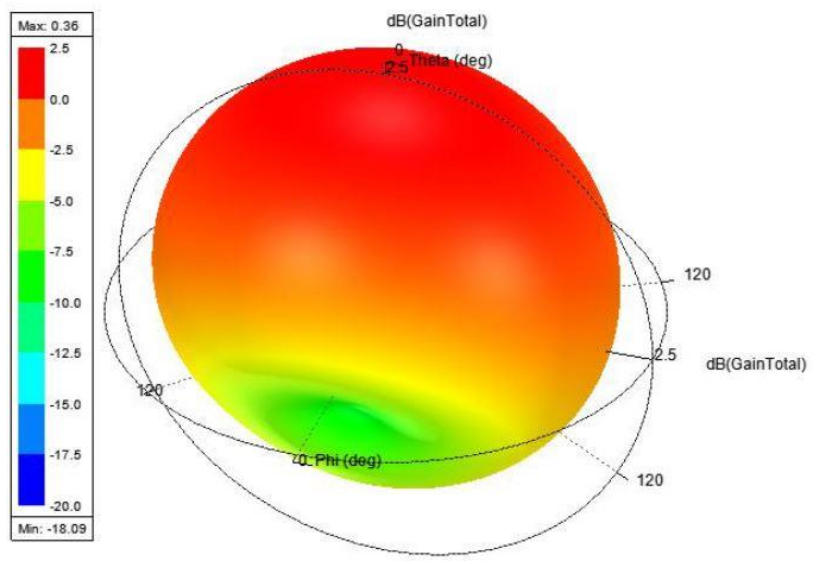

Fig. 4. Radiation Pattern of the proposed antenna

\section{CONCLUSION}

A miniaturized microstrip antenna with slots and high dielectric constant superstrate is presented for 900MHz RFID tags. The slot and two notches introduced are employed to extend the current path. The high-contrast dielectric superstrate loading helps in further miniaturization of the antenna and also helps in improving the radiation performance.

\section{REFERENCES}

[1] A. Holub and M. Polivka, "A Novel Microstrip Patch Antenna Miniaturization Technique: A Meanderly Folded Shorted-Patch Antenna," 2008 14th Conference on Microwave Techniques, Prague, 2008, pp. 1-4..

[2] F. Farzami, K. Forooraghi and M. Norooziarab, "Miniaturization of a Microstrip Antenna Using a Compact and Thin Magneto-Dielectric Substrate," in IEEE Antennas and Wireless Propagation Letters, vol. 10, pp. 1540-1542, 2011

[3] A. Roy, P. K. Choudhary, S. Anand, P. P. Sarkar and S. Bhunia, "A novel approach on miniaturization of microstrip patch antenna with loaded strips," International Conference on Electronics, Communication and Instrumentation (ICECI), Kolkata, 2014, pp. 1-4.

[4] Shao-Yi Chen, Hsi-Tseng Chou and Yi-Ling Chiu, "A size-reduced microstrip antenna for the applications of GPS signal reception," 2007 IEEE Antennas and Propagation Society International Symposium, Honolulu, HI, 2007, pp. 5443-5446.

[5] Hung Tien Nguyen, S. Noghanian and L. Shafai, "Microstrip patch miniaturization by slots loading," 2005 IEEE Antennas and Propagation Society International Symposium, Washington, DC, 2005, pp. 215-218 vol. $1 \mathrm{~B}$.

[6] J. M. Edwards and G. M. Rebeiz, "High-efficiency elliptical slot antennas with quartz superstrates for silicon RFICs," IEEE Trans. Antennas Propag., vol. 60, no. 11, pp. 5010-5020, Nov. 2012. 